\title{
Urocitograma y Parto *
}

\section{Drs. Guillermo López Escobar y César Mendoza Posada}

En comunicación preliminar a la III Convención de Ginecología y Obstetricia, reunida en Ibagué, revisamos la literatura en relación con el valor del Urocitograma como método para determinar la madurez placentaria y presentamos nuestras experiencias iniciales al respecto.

Durante más de dos años hemos estudiado un número mayor a 200 urocitogramas, de los cuales desechamos todos aquellos que por defecto de técnica, o por cualquiera otra causa, no han podido valorarse en forma correcta. El resto, comprende el material de esta presentación.

\section{METODOS EMPLEADOS}

Las pacientes fueron instruidas por uno de nosotros (G. L.), sobre la forma de recoger la muestra de orina y enviarla al Laboratorio; allí se recibió sin ningún conocimiento sobre época de embarazo y se procesó según la técnica corriente.

Las preparaciones se colorearon con el método de Papanicolau y en algunos casos con colorante de Shorr; como los resultados en cuanto a índice de eosinofilia fueron sensiblemente iguales con una y otra técnica, en placas duplicadas y de rutina, se utilizó la coloración de Papanicolau, por ser esta la corrientemente, usada en nuestro Laboratorio (C.M.P.).

Las placas fueron leidas conjuntamente (GL y $\mathrm{CM}$ ), sin conocer a quién pertenecían y solo después de examinarlas se cotejó el número de la placa con el nombre de la paciente y posteriormente con la época de embarazo. En esta forma pretendimos hasta el maximum evitar cuaiquier factor de error que por sugestión nos hiciera buscar explícitamente los cambios esperados.

En cada preparación se discriminó porcentualmente el número de células eosinofilas, superficiales, intermedias, cariopicnoticas, naviculares y otras tales como binucleadas, tipo post-partum etc.

En forma apreciativa se determinó la riqueza en células, su agrupación cantidad de leucocitos, moco, vitalidad celular, citolisis y alteraciones nucleares.

* Presentado al IV Congreso Colombiano de Obstetricia y Ginecología Barranquilla 1959. 
Con estos datos, se clasificaron los frotis en uno de tres tipos, según el criterio de los mexicanos (1-2-3) e independientemente de acuerdo al criterio de Lemberg y col., descrito para colpocitología. Comprendemos que estamos equiparando, talvez falsamente, citología vaginal con urocitología. Posteriorment, de acuerdo con la fecha del parto, se evaluó la realidad de los hallazgos. Brevemente recordaremos los criterios para determinar los distintos cuadros a pesar de que en la comunicación preliminar de hace dos años lo expusimos. Alvarez Bravo y González Ramos (1) describen: Cuadro Tipo I correspondiente a gestación inmadura para el parto, caracterizado por menos del $2 \%$ de células eosinótilas, $10 \%$ de picnóticas y $20 \%$ de superficiales, con abundantes naviculares, intermedias y profundas. Cuadro tipo II se presenta entre 3 y 7 días antes del parto; se caracteriza por eosinofilia del 2 al $10 \%$, cariopicnosis hasta un $30 \%$ y células superficiales hasta $40 \%$. Cuadro tipo III de madurez gravídica, se presenta dentro de los tres días anteriores al parto y se caracteriza por mas del $10 \%$ de eosinofilia, mas del $30 \%$ de cariopicnosis y mas del $40 \%$ de células superficiales, con ausencia o escasez de naviculares y fenómenos de desvitalización celular y alteraciones nucleares. Lemberg y col. (5) Encuentran sin significado los cambios en la eosinofilia y cariopicnosis y describen un tipo de embarazo avanzado no a término, embarazo cerca del termino y embarazo a termino. Las diférencias dependen de la descamación, vitalidad celular y cantidad de moco y leucocitos.

\section{MATERIAL UTILIZADO}

El estudio comprende 142 urocitogramas practicados en 46 pacientes, entre las cuales no se hizo discriminación de uni o multiparidad, edad, ni se fijaron fechas predeterminadas para el comienzo de toma de muestras. En general se empezó a practicar el urocitograma desde la 30 a 32 semana de embarazo pero en algunos casos se inició la serie mas precozmente. Los intervalos entre muestras no fueron regulares y se sujetaron a la facilidad de la paciente para enviar la muestra. El número de urocitogramas por paciente osciló entre 1 y 9.

\section{RESULTADOS}

Según el criterio de los autores mexicanos, en 53 preparaciones los hallazgos estuvieron acordes con la época de embarazo; lo cual da un índice de certeza de $37 \%$. Según los suizos en 61 casos $43 \%$. En los cuadros siguientes se resumen los resultados obtenidos tanto globales como discriminados en los diferentes estados: 


\begin{tabular}{|cccc|}
\hline & $\begin{array}{l}\text { UROCITOGRAMAS } \\
\text { (Clasificación mexicana) }\end{array}$ \\
Correspondencia & 53 casos & $37 \%$ \\
\hline No correspondencia & $89 \quad "$ & $63 \%$ \\
\hline
\end{tabular}

\begin{tabular}{|lrlll|}
\hline TIPO I 33 casos & & & \\
Correspondencia & 28 &, & $85 \%$ \\
No correspondencia & 5 &, & $15 \%$ \\
\hline TIPO II 93 casos & & & \\
Correspondencia & 19 &, & $24 \%$ \\
No correspondencia & 74 & & $76 \%$ \\
\hline TIPO III 16 casos & & & & \\
\hline Correspondencia & & & \\
No correspondencia & 10 &, & $38 \%$ \\
\hline
\end{tabular}

\begin{tabular}{|c|c|c|c|c|}
\hline \multicolumn{5}{|c|}{$\begin{array}{l}\text { UROCITOGRAMAS } \\
\text { (Clasificación suiza) }\end{array}$} \\
\hline Correspondencia & 61 & $\operatorname{asos} 4$ & $43 \%$ & \\
\hline No correspondencia & & $"$ & $57 \%$ & \\
\hline TIPO I 79 casos & & & & \\
\hline $\begin{array}{l}\text { Correspondencia } \\
\text { No correspondencia }\end{array}$ & $\begin{array}{l}65 \\
14\end{array}$ & $\begin{array}{ll}" & 6 \\
" & 1\end{array}$ & $\begin{array}{l}63 \% \\
17 \%\end{array}$ & \\
\hline $\begin{array}{l}\text { TIPO II } 50 \text { casos } \\
\text { Correspondencia }\end{array}$ & 13 & $" 2$ & $26 \%$ & \\
\hline No correspondencia & 37 & $" 7$ & $74 \%$ & \\
\hline $\begin{array}{l}\text { TIPO III } 13 \text { casos } \\
\text { Correspondencia }\end{array}$ & 3 & $" 2$ & $23 \%$ & \\
\hline No correspondencia & 10 & $" 6$ & $67 \%$ & \\
\hline
\end{tabular}




\section{COMENTARIOS}

De lo anterior se deduce que ei mayor índice de certeza, tanto utilizando uno como otro criterio, corresponde a los estados I (85\% según criterios mexicanos) (63\% según los suizos).

En contraposición los mayores errores se encuentran en el estado II mexicano $76 \%$, suizo $74 \%$ ) que es precisamente el que observamos mas frecuentemente según el criterio mexicano (93 casos, $65 \%$ ) y el segundo en frecuencia según los suizos.

El análisis de los hallazgos combinando los dos criterios no aporta mejores datos ya que no hay un patrón de correspondencia esta dísticamente valedero.

En algunos casos se revisaron las placas después de conocida la fecha del parto para comprobar si conociendo ésta, podría el observador pensar que había error en la valoración del grado correspondiente y aún así, conociendo el resultado a posteriori, no creimos que honestamente pudiéramos cambiar la catalogación hecha en tales urocitogramas.

$\mathrm{Al}$ observar el cuadro de resultados llama la atención el alto porcentaje de certeza en los estados I, pero debemos recordar que la toma de muestras fue iniciada en la mayoría de las pacientes desde ocho a diez semanas antes de la fecha de confinamiento. Siendo asi que con el tipo de clasificación suiza, no importa la cercanía del parto, el mayor número de placas (79) dan la pariencia de grado I, por razones de azar debe el observador acertar en un buen número de casos.

En cambio es de subrayar que de 10 a 40 días antes de la fecha del parto en varias pacientes encontramos un aspecto típico de grado III, es decir, de parto inminente, fenómeno que nosotros denominamos época de transición pues en los urocitogramas posteriores el aspecto cambia totalmente para volver a dar la apariencia de una placenta inmadura, es decir un grado I o a veces II. Este fenómeno puede constituír una de las grandes causas de error para el citólogo.

Esta época es pasajera, pero no constante, de ahí lo aleatorio de los resultados.

En tres casos, en que el envío de la muestra coincidió con el mismo día del parto espontáneo, los resultados de las placas leídas en la forma anotada en la metodología del trabajo, es decir sin conocimiento previo de la fecha de éste fueron los siguientes: dos casos los clasificamos como Tipo III, tanto con criterio mexicano, como suizo y uno como Tipo II, por ambos criterios. Esto parecería muy acertado, pero desgraciadamente como lo muestra el cuadro 
previamente presentado, tuvimos en 16 casos catalogados como Tipo III, según criterio mexicano, 10 falsos; y sobre 13 casos, con el criterio suizo, 10 falsos.

Podemos afirmar, que sí hay cambios en el urocitograma durante la última semana del embarazo, pero que éstos cambios no permiten de modo absoluto darle al obstetra la seguridad para que pueda practicar en forma rutinaria lo que llama Alvarez "El desencadenamiento inminentte del parto". Pues el Urocitograma no es en nuestra experiencia, un criterio que permita establecer en materia de horas ó de días, cuando se va a producir el parto. Aunque puede dar un índice a grosso modo de la fecha del parto, sinembargo, no puede equipararse al dato clínico de madurez del cuello, que aunque tampoco es exacto, en multitud de casos orienta mejor al tocólogo, que el urocitograma mismo. Es claro que la combinación de los dos métodos puede darnos mayor seguridad, pero sin llegar a constituír el ideal que nos permita inducir sistemáticamente, sin peligro, a todas las pacientas. Creemos que en manos de un buen obsttetra estas inducciones llamadas de "desencadenamiento inminente del parto" muchas veces son forzadas y que naturalmente es la pericia del tocólogo la que hace que no se produzcan complicaciones. Pero estamos convencidos de que todavía no podemos predecir con exactitud de e hora o días la fecha precisa del parto y por lo tanto no podemos recomendar a priori la generalización de inducciones a todas las pacientes,con base en estos métodos.

\section{BIBLIOGRAFIA}

1 ALVAREZ BRAVO A. y GONZALEZ RAMOS. El urocitograma al final del embarazo. Gynec. y Obst. de México. V. XI May. Jun 1956, página 231.

2 ALVAREZ BRAVO A. y col. Vol.- Inducción electiva del parto. Gynec y Obst. de México col. Vol. V. XI Mayo, jun 1956, página 209.

3 ALVAREZ BRAVO y col.- La citología vaginal y la citología del sedimento urinario en el embarazo. Revista colombiana de Obstetricia y Gynec. Vol № 2. Marzo, Abril 1957, página 77 .

4 LEMBERG) SIGFRIED S. STAMM O.- Citologgie vaginale a la la Grossesse. Neuvelle Methode de Diagnostic de la Grossesse a terme. Exposition Scientifique Congres Int. de Ginec. et Obst. Ginebra 1954. Editado por Sandoz S. A. Basilea. Página. 62.

5 LEMBERG SIEFRIED S. STAMM O. y WATWVILLE H. Le frottis vaginal aux differents stades de la grossese normal et pathologique La Presse Medicale 63 № 76 Nov. 1955 pág. 1958.

6 ESCOBAR G y MENDOZA C. Urocitograma y Parto Revista Colombiana de Ginecol y Obst. 9:500 Sep. 1958. 